\title{
Monocapas de ácidos grasos. I. Ácido esteárico sobre disoluciones acuosas de etanol.
}

\author{
Por J. M. Rodríguez Patino, J. de la Fuente Feria y C. Gómez Herrera \\ Departamento de Ingeniería Química. Facultad de Química. \\ Universidad de Sevilla. 41012 - Sevilla (España)
}

\section{RESUMEN}

Monocapas de ácidos grasos. I. Acido esteárico sobre disoluciones acuosas de etanol.

Se ha estudiado la estructura y estabilidad de monocapas de ácido esteárico esparcidas sobre disoluciones acuosas que contienen etanol. Las experiencias se han realizado en una balanza de superficie comercial tipo Langmuir y se ha operado en condiciones isotérmicas.

Las monocapas presentan estructuras de tipo sólida o de liquido condensado en función de la concentración de etanol en la subfase. La estructura que adopta la monocapa es prácticamente independiente de la temperatura. La pérdida de moléculas de ácido esteárico de la monocapa por disolución en la subfase se incrementa a los valores más elevados de temperatura y de concentración de etanol.

PALABRAS-CLAVE: Acido esteárico - Balanza de superficie Estabilidad de emulsión - Monocapa.

\section{SUMMARY}

Monolayers of fatty acids. I. Stearic acid on aqueous solutions of ethanol.

The structure and stability of fatty acid monolayers spread on aqueous solutions with ethanol has been studied. The experiments were carried out isothermically using a commercial Langmuir balance (Lauda).

Monolayers exhibit solid or condensed liquid structure depending of the ethanol concentration in the subphase. Temperature has not a significant influence on the monolayer. Structure there is a loss of stearic acid film through solution into the adjacent subphase. This loss is increased with the temperature and ethanol concentration in the subphase.

KEY-WORDS: Emulsion stability - Monolayer - Stearic acid Surface balance.

\section{INTRODUCCION}

Las emulsiones alimentarias son termodinámicamente inestables a causa de su elevada área interfacial. Para su conservación temporal se requiere la incorporación de productos anfifílicos que contengan en una misma molécula, o en un mismo ión, funciones hidrófilas y funciones hidrófobas (1-3). Un amplio porcentaje de estos aditivos alimentarios lo constituyen lípidos polares, entre los que caben destacar a los fosfolípidos, monoglicéricos y ésteres de ácidos grasos de cadena larga con polioles (4-7).
En la utilización industrial de un emulsionante alimentario y en las cualidades que confiere al producto acabado, es necesario conocer la estructura de la película de lípido en las interfases aceite-agua o aire-agua. En estos sistemas las interacciones entre las moléculas que forman la monocapa se han relacionado con la viscosidad y elasticidad de la película superficial (8) (9) y con la estabilidad de emulsiones y espumas (10-12). Además, se pueden establecer relaciones entre la estabilidad de una emulsión y las características estructurales de las monocapas de lipidos en las interfases aceite-agua y aire-agua (6) (12-14), y también entre la formación de monocapas condensadas y la asociación de los lípidos en el seno de las disoluciones (6) (15) (16). Estas dependencias son interesantes ya que desde un punto de vista experimental es más fácil estudiar las monocapas de lípidos en la interfase aire-agua que en la interfase aceite-agua (11) (12).

Aunque se han propuesto numerosos modelos teóricos para explicar las características de las monocapas en la interfase aire-agua, según las interacciones entre las moléculas (17-19), son necesarios posteriores desarrollos más elaborados para lo cual ha de disponerse de nuevos y más fiables datos experimentales de monocapas esparcidas sobre medios acuosos de diversas características superficiales. Esto es especialmente útil para predecir las formulaciones óptimas de emulsiones y espumas (11).

El objetivo de este trabajo ha consistido en la determinación de las características estructurales y de la estabilidad de monocapas de ácido esteárico, constituyente de ésteres de ácidos grasos y polioles utilizados como aditivos alimentarios, esparcidas sobre disoluciones acuosas de etanol, en función de la temperatura y de la composición del medio acuoso.

\section{MATERIALES $Y$ METODO}

Las isotermas (curvas presión de esparcimiento, $\pi$, frente al área por molécula de ácido esteárico, A) se han obtenido en una balanza de superficie comer- 
cial tipo Langmuir (Messgerate-Werk Dr. R. Wobser KG, Modelo Filwaage FW-1 serie CO3001, Lauda) equipada con un sistema de medida continuo $y$ registrador. La temperatura de la bandeja que contiene la monocapa y la subfase se mantiene constante durante cada operación mediante un termostato (Lauda, Modelo K2R Electronic), con una sensibilidad en el control de temperatura de $\pm 0^{\prime} 01-0^{\prime} 03^{\circ} \mathrm{C}$.

Los productos usados son de calidad analítica y no han sido sometidos a purificación posterior: ácido esteárico (Merck, Cat. 800673) y etanol (Merck, Cat. 971). El agua fue purificada mediante una unidad de filtración Millipore (Mille $Q^{T M}$ ). El ácido esteárico fue esparcido sobre la subfase previa disolución del mismo en benceno (Merck, Cat. 1783). La actividad superficial de los componentes de la subfase y del portador del ácido esteárico fue comprobada experimentalmente antes de registrar las isotermas correspondientes.

Al iniciar cada experiencia se calibra la balanza. La sustancia formadora de monocapa se esparce sobre la subfase mediante una jeringa micrométrica y su masa se determina por pesada en balanza analítica. En función del número de moléculas de lípido esparcidas se fija un valor en la unidad de control que relativiza la superficie ocupada por molécula, $R$. La velocidad de compresión de la monocapa fue de 3'3 $\mathrm{cm} / \mathrm{min}$. Ambos parámetros se mantienen constantes durante la realización de cada operación.

\section{RESULTADOS Y DISCUSION}

\subsection{Monocapas de ácido esteárico sobre agua} desionizada

Las experiencias con ácido esteárico se inician con el producto esparcido sobre una subfase constituida por agua desionizada que servirá de referencia para aquellos sistemas en los que en la subfase se incluyan moléculas semejantes a las presentes en formulaciones alimentarias. Los resultados obtenidos referidos a la influencia de la temperatura sobre las características de la monocapa se resumen en la figura 1. Es observable que:

* La monocapa presenta un polimorfismo caracterís-

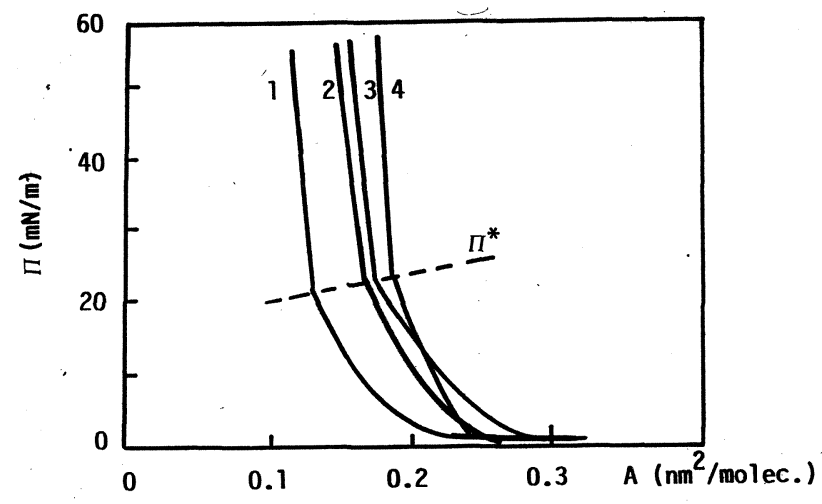

Figura 1

Isotermas de compresión presión superficial-área por molécula de ácido esteárico. Subfase: Agua desionizada. Temperaturas. $1: 5^{\circ} \mathrm{C}, 2: 10^{\circ} \mathrm{C}, 3: 15^{\circ} \mathrm{C}, 4: 20$ $40^{\circ} \mathrm{C}$.

tico de una estructura líquido condensada (LC) que se transforma en sólida (S) al disminuir el área disponible para las moléculas que la constituye.

* La presión característica $\left(\pi^{*}\right)$ a la que se produce la transición $\mathrm{LC}=\mathrm{S}$, depende de la temperatura a la cual se realiza la experiencia. El valor de la presión característica se incrementa al aumentar la temperatura, según se expone en la tabla 1 .

* La temperatura no ejerce influencia significativa sobre las características estructurales de la monocapa, si bien su aumento produce un desplazamiento de las isotermas hacia mayores solicitudes de área. Es decir, la monocapa mantiene sus estructuras de sólido o de líquido condensado pero ocupando mayores áreas por molécula al incrementarse la temperatura. Los valores del área por molécula en función de la temperatura deducidos por extrapolación de las isotermas correspondientes a las monocapas con estructura sólida $\left(A_{\text {lim }}\right)$ se incluyen en la tabla I.

* Las solicitudes de área por las moléculas de la monocapa son independientes de la temperatura para valores superiores a $20^{\circ} \mathrm{C}$, lo cual puede atribuirse al aumento de la solubilidad de la monocapa en la subfase (pérdida de moléculas de ácido esteárico

\begin{tabular}{|c|c|c|c|c|c|c|}
\hline \multirow[t]{2}{*}{ TABLA I } & \multicolumn{6}{|c|}{$\begin{array}{l}\text { Area IImite y presion caracteristica de la transicior } \\
\text { LC }=\mathrm{s} \text { en funcion de la temperatura de monocapas de } \\
\text { acido estearico esparcidas sobre agua desionizada. }\end{array}$} \\
\hline & $\Gamma(8 C)$ & 5 & 10 & 15 & 20 & 40 \\
\hline \multicolumn{2}{|c|}{$\pi^{*}(\mathrm{mN} / \mathrm{m})$} & $21 \cdot 2$ & $22^{\prime} 0$ & $23^{\prime} 5$ & $24^{\prime} 0$ & $24^{\prime} 0$ \\
\hline \multicolumn{2}{|c|}{$A_{11 m}\left(\mathrm{~nm}^{2} / \mathrm{mol} \varepsilon \mathrm{c}\right)$} & 0.142 & $0^{\prime} 176$ & 0.188 & 0.197 & 0.197 \\
\hline
\end{tabular}


por disolución en la subfase) debido al incremento de la temperatura, o bien a una pérdida parcial de la misma como consecuencia del arrastre de las moléculas que forman la monocapa ocasionada por la evaporación de la subfase a las temperaturas superiores. Es conveniente recordar a tal efecto que el valor del parámetro $R$ que relativiza la solicitud de área por molécula de forma automática mediante la unidad de control se mantiene en un valor constante para cada experiencia, independientemente de la temperatura a la cual se realiza la compresión.

3.2. Monocapas de ácido esteárico sobre disoluciones de etanol en la subfase

El estudio de la influencia de la concentración de etanol en la subfase sobre las características de las monocapas de ácido esteárico se ha realizado en cinco disoluciones con concentraciones de etanol comprendidas entre 0 ' 1 y $2 \mathrm{~mol} / \mathrm{L}$.

Disolución acuosa de etanol a 0 '1 moll como subfase.

Los resultados obtenidos se representan en la figura 2. Para el análisis de los resultados es conveniente indicar que la primera isoterma se registra a $20^{\circ} \mathrm{C}$, a continuación se realiza la experiencia a la temperátura de $40^{\circ} \mathrm{C} . y$, finalmente, se efectúan distintas compresiones a $5^{\circ} \mathrm{C}$. A partir de las isotermas registradas se obtiene, por extrapolación del segmento recto característico de la estructura sólida de la monocapa, las áreas por molécula que se exponen en la tabla II. De los resultados obtenidos se deduce lo siguiente:

* La monocapa posee estructura de líquido condensado que se transforma en sólida al aumentar la presión de compresión o disminuir el área disponi-

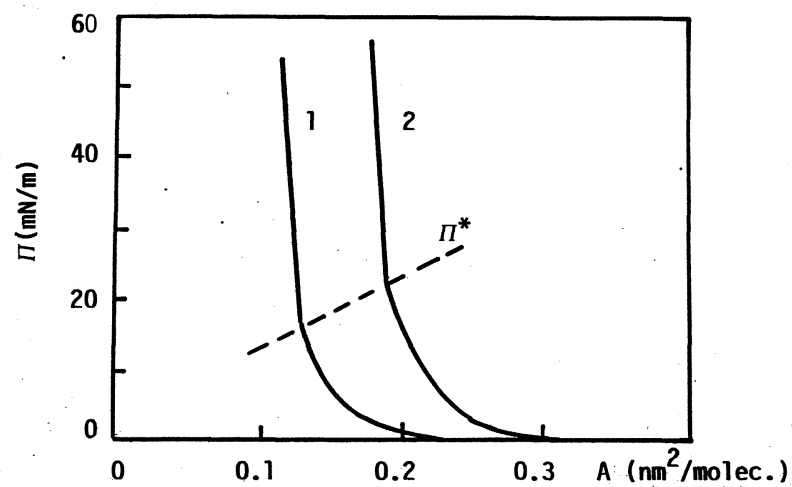

Figura 2

Isotermas de compresión presión superficial-área por molécula de ácido esteárico. Subfase: Disolución acuosa de etanol de $0.1 \mathrm{~mol} / \mathrm{L}$. Temperaturas. $1: 5^{\circ} \mathrm{C}, 2$ : $25-40^{\circ} \mathrm{C}$.

ble. La presión característica $\left(\pi^{\star}\right)$ a la cual se produce la citada transición aumenta al incrementarse la temperatura desde $5^{\circ} \mathrm{C}$ hasta $20^{\circ} \mathrm{C}$.

* La estructura y el área por molécula de la monocapa son independientes de la temperatura cuando esta supera los $20^{\circ} \mathrm{C}$. La coincidencia de las isotermas de compresión que se obtienen en el intervalo comprendido entre 20 y $40^{\circ} \mathrm{C}$ puede deberse a la disolución del ácido esteárico de la monocapa en la subfase en una proporción tal que el previsible aumento del requerimiento de área con la temperatura se compense por la existencia de menos moléculas en la superficie.

* Al reducirse la temperatura del sistema de $20^{\circ} \mathrm{C}$ a $5^{\circ} \mathrm{C}$ disminuye el área ocupada por cada molécula, si bien se mantiene la estructura de la monocapa. Tras la realización de compresiones sucesivas a la citada temperatura se observa una disminución progresiva del área por molécula ocupada por la monocapa. El hecho de que la reducción del área por

\begin{tabular}{|c|c|c|c|}
\hline TABLA II & $\begin{array}{l}\text { Sol1c1tud } \\
\text { estear1co } \\
0.1 \mathrm{~mol} / \mathrm{L} .\end{array}$ & $\begin{array}{l}\text { de area por moldev } \\
\text { esparcidas sobre }\end{array}$ & $\begin{array}{l}\text { la de monocapas de acido } \\
\text { disolución de etanol, }\end{array}$ \\
\hline & & $T(\Omega C)$ & $A\left(\mathrm{~nm}^{2} / \mathrm{mol} \mathrm{C}\right)$ \\
\hline 10 & Compres $18 \mathrm{n}$ & 20 & 0.202 \\
\hline 22 & Compresion & 40 & 0.202 \\
\hline 12 & Compres18n & 5 & 0.170 \\
\hline 2 & Compres $18 n$ & B & 0.150 \\
\hline & Compres18n & 5 & 0.134 \\
\hline 40 & Compres18n & 5 & 0.134 \\
\hline 58 & Compres $18 n$ & 5 & 0.134 \\
\hline
\end{tabular}




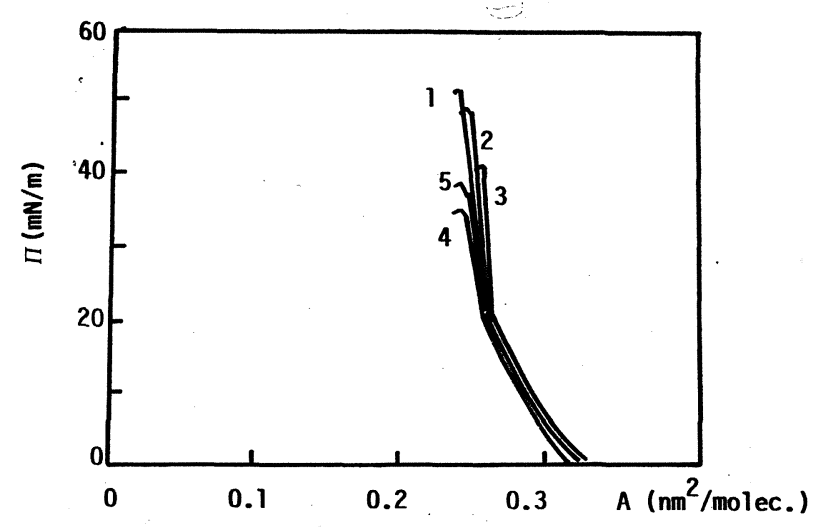

Figura 3

Isotermas de compresión presión superficial-área por molécula de ácido esteárico. Subfase: Disolución acuosa de etanol de $0.2 \mathrm{~mol} / \mathrm{L}$. Temperaturas. $1: 5^{\circ} \mathrm{C}$, 2: $10^{\circ} \mathrm{C}, 3: 20^{\circ} \mathrm{C}, 4: 30^{\circ} \mathrm{C}, 5: 40^{\circ} \mathrm{C}$

molécula tienda a un valor constante a partir de la cuarta compresión a la que se somete la monocapa parece indicar que la citada dependencia está relacionada con la tendencia del sistema a alcanzar las condiciones estacionarias. La disminución del área ocupada por molécula a la temperatura inferior es de difícil justificación, si no se admite la existencia de una disolución previa de las moléculas de la monocapa a las mayores temperaturas.

Disolución acuosa de etanol a 0'2 moll como subfase.

Las monocapas de ácido esteárico esparcidas sobre una disolución 0'2 mol/L de etanol se caracte-

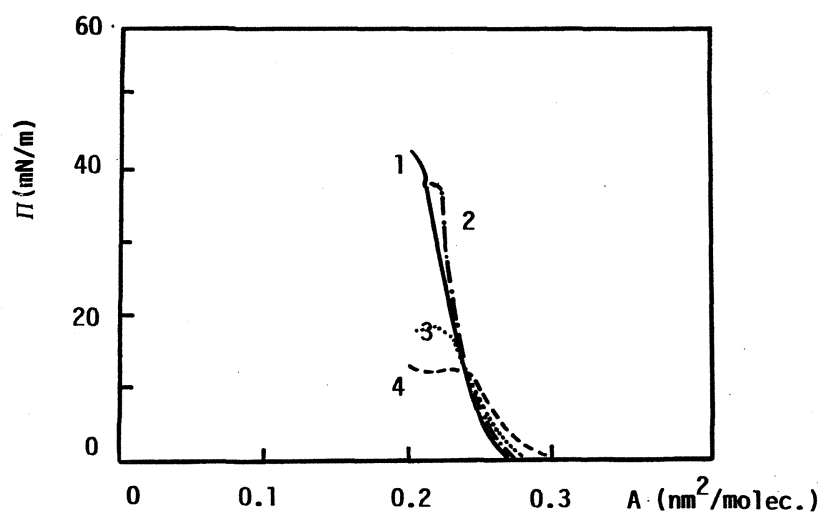

Figura 4

Isotermas de compresión presión superficial-área por molécula de ácido esteárico. Subfase: Disolución acuosa de etanol de $0.5 \mathrm{~mol} / \mathrm{L}$. Temperaturas. $1: 5^{\circ} \mathrm{C}$, 2: $10^{\circ} \mathrm{C}, 3: 25^{\circ} \mathrm{C}$ (primera compresión), 4: $25^{\circ} \mathrm{C}$ (segunda compresión).

rizan por poseer una estructura y una solicitud de área por molécula que son independientes de la temperatura en el intervalo comprendido entre 5 y $40^{\circ} \mathrm{C}$, según se deduce de las isotermas registradas en la figura 3. Estos resultados parecen confirmar las razones expuestas en el apartado correspondiente a la monocapa de ácido esteárico esparcida sobre disolución de etanol a $0^{\prime} 1 \mathrm{~mol} / \mathrm{L}$ y sometidas a compresiones continuas, a la temperatura de $5^{\circ} \mathrm{C}$. En efecto, al realizarse en este caso las experiencias en el orden indicado en la figura, de temperaturas crecientes, y transcurrido un período de tiempo entre cada compresión, las isotermas registradas a cada temperatura son coincidentes. La independencia entre las isotermas

\begin{tabular}{|c|c|c|c|c|c|}
\hline \multirow{2}{*}{$\begin{array}{l}\text { TABLAIII Presion de } \\
\text { esparcidas }\end{array}$} & $\begin{array}{l}\text { colar } \\
\text { sobre } 0\end{array}$ & \multicolumn{2}{|c|}{$\begin{array}{l}\text { de monocapa } \\
\text { olucion acuose }\end{array}$} & $\begin{array}{l}\text { ac1d } \\
\text { etanol }\end{array}$ & $\begin{array}{l}\text { steár } 1 \mathrm{co} \\
\mathrm{mol} / \mathrm{L} .\end{array}$ \\
\hline & 5 & 10 & 20 & 25 & 40 \\
\hline$\pi_{C}(\mathrm{mN} / \mathrm{m})$ & $52^{\prime} 1$ & $54^{\prime} 0$ & $44: 4$ & $35^{\prime} 2$ & $39 \cdot 1$ \\
\hline
\end{tabular}

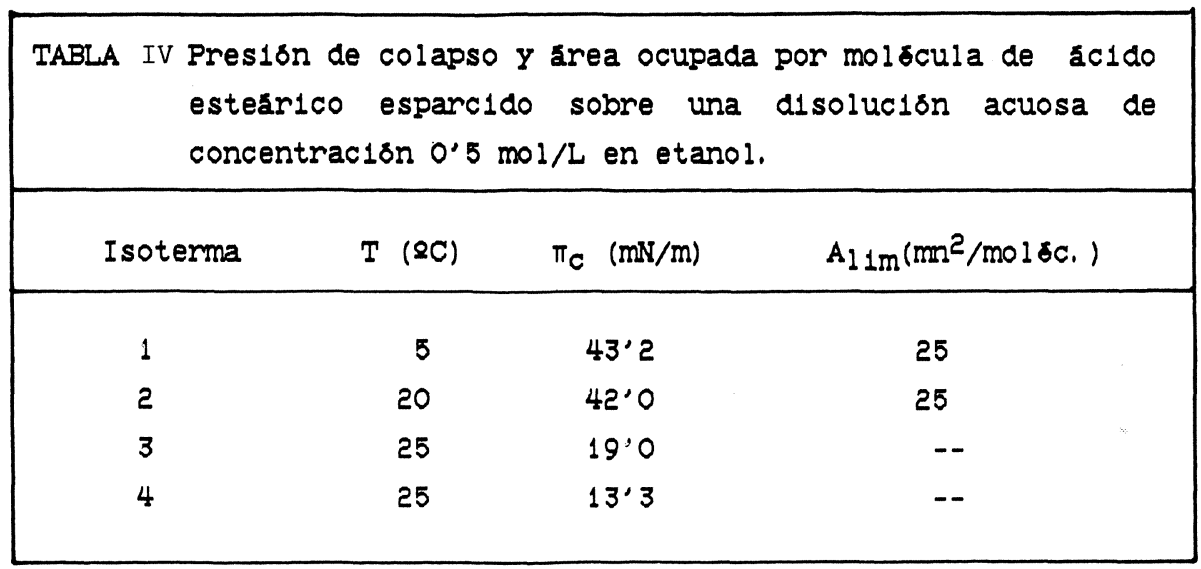


obtenidas y la temperatura puede atribuirse a la disolución de las moléculas de la monocapa en la subfase, que se incrementa al aumentar la temperatura.

Un aumento de temperatura modifica la presión de colapso $\left(\pi_{c}\right)$ de la monocapa, según se expone en la tabla III. Estos resultados son consistentes con la afirmación de existencia de inestabilidad de la monocapa por disolución, de sus moléculas en la subfase en las condiciones de experimentación. En efecto, la existencia de una elevada presión de colapso se ha asociado a un incremento de las fuerzas de cohesión de Van der Waals entre las moléculas de la monocapa (20) (21) un hecho que determina cuándo una molécula puede formar una monocapa insoluble (22) (23).

Disolución acuosa de etanol a 0'5 moll como subfase.

En la figura 4 se resumen los resultados obtenidos al comprimir una monocapa de ácido esteárico esparcido sobre una disolución acuosa de etanol de concentración 0'5 mol/L. Su estructura es prácticamente independiente de la temperatura, existiendo una transición líquido condensada $=$ sólida al aumentar la presión ejercida sobre la misma. En estas circunstancias la temperatura incide sobre la presión de colapso de la monocapa disminuyendo su valor al aumentar la temperatura, según se muestra en los resultados incluidos en la tabla IV. Finalmente, tras la realización de dos compresiones consecutivas a la temperatura de $25^{\circ} \mathrm{C}$ se reduce la presión de colapso.

Los resultados representados en la figura 4 refuerzan la idea de la importancia de la solubilidad de la monocapa. En efecto, es observable en estas circunstancias que al aumentar la temperatura se incrementa la solubilidad de la monocapa manteniéndose su estructura. Es decir, la esperable expansión que la monocapa debe experimentar con la temperatura se compensa con la existencia de la disolución de algunas de sus moléculas y las isotermas se registran con idéntica solicitud de área por molécula, independientemente de la temperatura a la cual se realiza la compresión. Esta idea es coincidente con los datos expuestos en la tabla IV sobre la dépendencia entre temperatura y la presión de colapso. Por lo tanto, la reducción en la presión de colapso, tras compresiones sucesivas a la temperatura de $25^{\circ} \mathrm{C}$ puede ser la consecuencia de la existencia de un período transitorio tras un proceso de disolución previa, un fenómeno similar al discutido previamente al esparcir el mismo ácido graso sobre disolución acuosa de etanol de concentración 0'1 M. Este comportamiento es semejante al de la mayoría de las monocapas de lípidos esparcidas sobre agua en las cuales se ha comprobado la existencia de disolución (24-26).
Disolución acuosa de etanol a $1 \mathrm{~mol} / \mathrm{L}$ como subfase.

La isoterma de compresión presión superficial-área de una monocapa de ácido esteárico esparcida sobre disolución acuosa de etanol de concentración $1 \mathrm{~mol} /$ $\mathrm{L}$ a la temperatura de $20^{\circ} \mathrm{C}$ se representa en la figura 5. Al igual que ocurre con los resultados experimentales discutidos en apartados anteriores, es lógico pensar en la existencia de disolución de moléculas de la monocapa en la subfase de concentración $1 \mathrm{~mol} /$ $L$ de etanol.

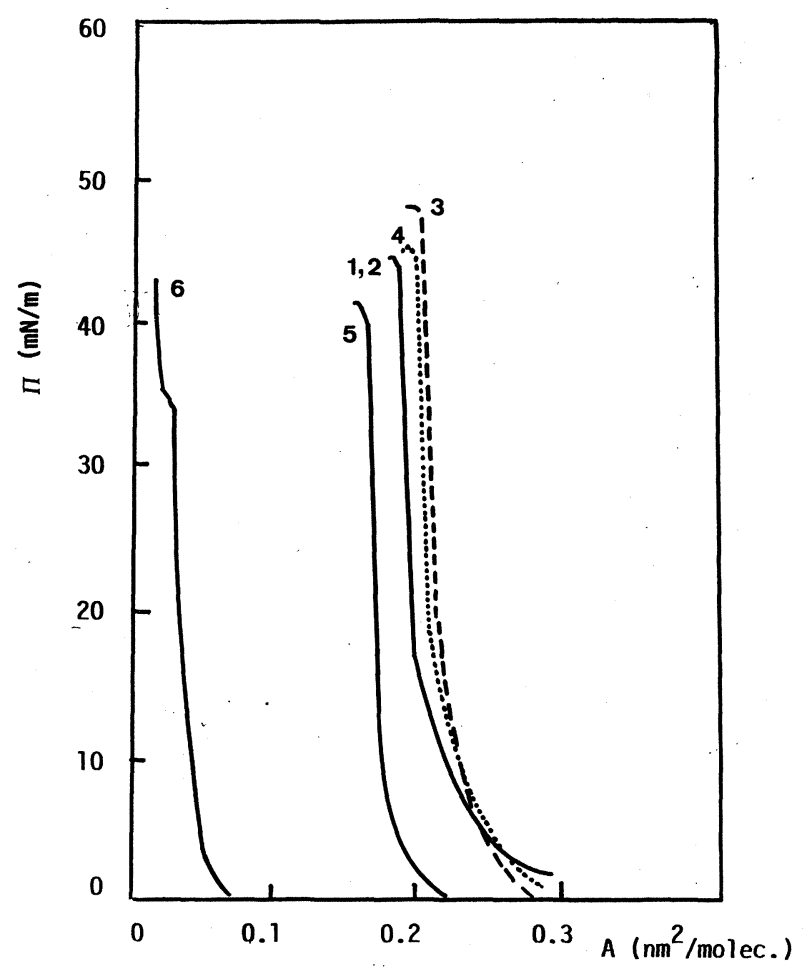

Figura 5

Isotermas de compresión presión superficial-área por molécula de ácido esteárico a $20^{\circ} \mathrm{C}$. Subfase: Disoluciones acuosas de etanol. 1: $0 \mathrm{~mol} / \mathrm{L}, 2: 0.1 \mathrm{~mol} /$ L, 3: $0.2 \mathrm{~mol} / \mathrm{L}, 4: 0.5 \mathrm{~mol} / \mathrm{L}, 5: 1 \mathrm{~mol} / \mathrm{L}, 6: 2 \mathrm{~mol} / \mathrm{L}$.

Disolución acuosa de etanol a 2 mol/L como subfase.

En la figura 5 se incluye la isoterma correspondiente a la primera compresión realizada sobre una monocapa de ácido esteárico esparcido sobre una disolución de etanol en agua de concentración $2 \mathrm{~mol} /$ $L$ en soluto.

Es interesante destacar la significativa reducción en el área por molécula al comparar esta experiencia con las discutidas en apartados anteriores, referidas a disoluciones con menor contenido de etanol en la subfase. Esta reducción en la solicitud de área por molécula ha de atribuirse necesariamente a un 
proceso de disolución de moléculas de la monocapa, incluso a las menores temperaturas. En efecto, de la misma isoterma se observa que la estructura de la monocapa es semejante a la deducida de las experiencias comentadas en apartados anteriores, con una de sólido o de líquido condensado, según la presión ejercida sobre la monocapa. Para estas condiciones la influencia de la temperatura se concreta en una reducción de la presión de colapso de la monocapa con el aumento de temperatura.

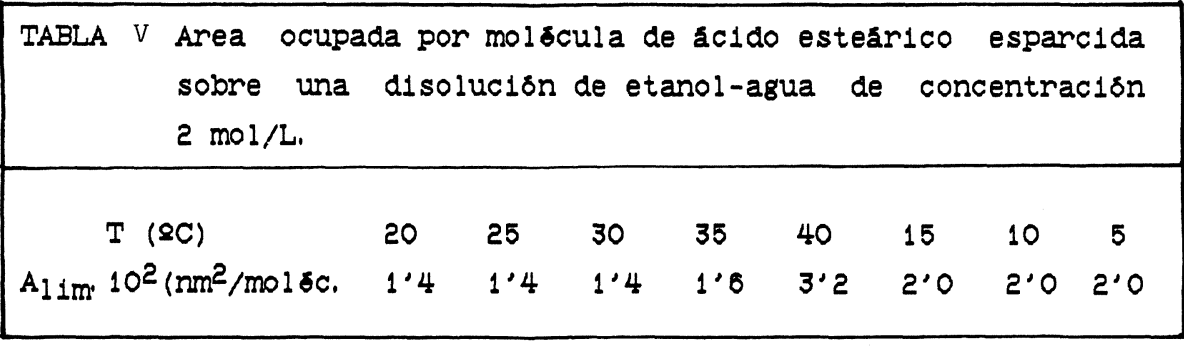

presión de colapso próxima a los $50 \mathrm{mN} / \mathrm{m}$, si bien es de destacar la existencia, a esta temperatura, de una meseta a la presión de $32 \mathrm{mN} / \mathrm{m}$ que separa la existencia de dos configuraciones distintas de la monocapa. Es posible, no obstante que el registro de esa meseta se deba a un solapamiento de las isotermas correspondientes a la monocapa y a la subfase. Se ha comprobado experimentalmente que para bajas solicitudes de área por molécula, cuando ambas barreras, la móvil y la de medición, están próximas, la subfase presenta una actividad superficial propia (27).

Las isotermas correspondientes a las compresiones realizadas sobre la monocapa en el intervalo comprendido entre 5 y $40^{\circ} \mathrm{C}$ no se incluyen en la figura debido a la proximidad de sus registros al eje de abscisas, si bien las solicitudes de área por molécula deducidas por extrapolación de los mismos se exponen en la tabla $V$, debido a que en el citado intervalo
En la figura 5 se representan las isotermas correspondientes a monocapas de ácido esteárico esparcido sobre disoluciones alcohólicas con distintas concentraciones de soluto a la temperatura de $20^{\circ} \mathrm{C}$. En estas circunstancias:

* la transformación sólido $\rightarrow$ líquido condensado se favorece con el aumento de la concentración de etanol.

* al aumentar la concentración de etanol en la subfase se reduce la presión de colapso de la monocapa, aunque el valor de área ocupada por cada molécula es prácticamente insensible a la citada variación, según se expone en la tabla VI. Los resultados obtenidos con una concentración $2 \mathrm{M}$ de etanol en la subfase constituye una excepción en el comportamiento antes comentado.

En la figura 6 se representa la dependencia de la concentración de etanol en la subfase y de la temperatura sobre el área ocupado por cada molécula

\begin{tabular}{|c|c|c|c|c|c|c|}
\hline \multicolumn{7}{|c|}{$\begin{array}{l}\text { TABLA VI Presion de colapso y area ocupada por molocula de acido } \\
\text { estearico esparcida sobre disoluciones de etanol a 208c. }\end{array}$} \\
\hline Etanol (mol/L) & 0 & 0.1 & 0.2 & 0.5 & 1 & 2 \\
\hline$\pi_{e}(m N / m)$ & & 60 & 49 & 42 & $37^{\prime} 8$ & 33 \\
\hline$A_{11 m}\left(\mathrm{~nm}^{2} / \operatorname{mol} 18 \mathrm{c}\right)$ & 0.202 & 0.200 & 0.218 & 0.216 & 0.210 & 0.043 \\
\hline
\end{tabular}

de temperaturas, la monocapa mantiene una estructura sólida. El orden de realización de las compresiones coinciden con el indicado en la citada tabla.

Dicusión global de resultados con etanol como'subfase.

De las experiencias realizadas se deduce una práctica independencia entre las estructuras de la monocapa y la temperatura, cualquiera que sea la concentración de etanol en la subfase. Dichas estructuras, según lo comentado en los apartados correspondientes son coincidentes con las configuraciones de ácido esteárico correspondiente a su estructura sólida. Según puede observarse la influencia de la concentración de etanol en la subfase es cuantitativamente superior a la ejercida por la temperatura. Además, y debido a que no es esperable que la estructura que adoptan las moléculas de ácido esteárico en la monocapa sea más compacta que la existente en su estructura cristalina (28), los valores del área límite de la monocapa inferiores al área ocupada por los átomos en dicho cristal son concordantes con la opinión de existencia de un proceso de disolución 


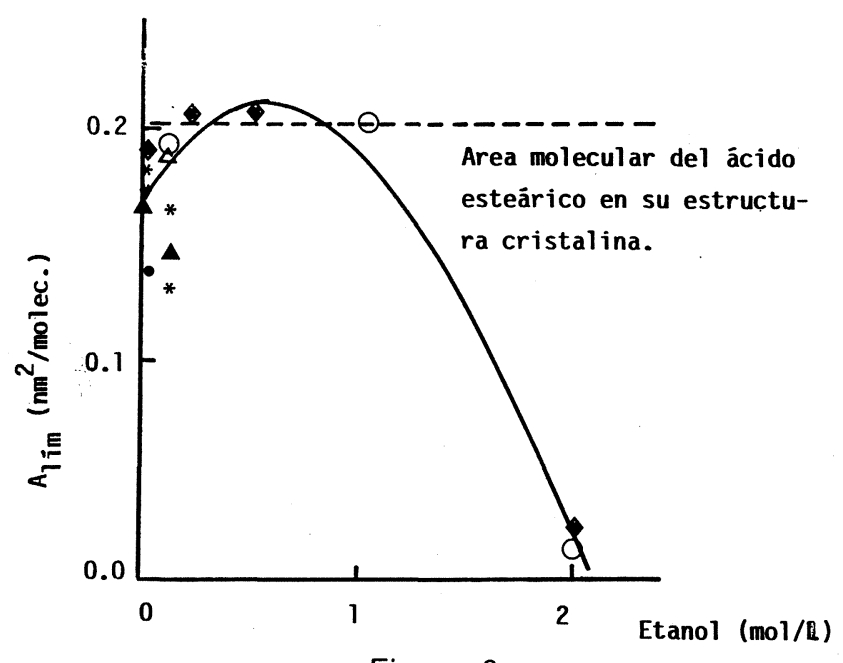

Figura 6

Influencia de la temperatura y de la concentración de etanol en la subfase sobre el área límite de monocapas de ácido esteárico.

\begin{tabular}{|ccccccc|}
\hline$T(\stackrel{\circ}{ } \mathrm{C})$ & 5 & 10 & 15 & 20 & 30 & 40 \\
CLAVE & $\bullet$ & $\Delta$ & $*$ & 0 & $\Delta$ & $\diamond$ \\
\hline
\end{tabular}

de moléculas de ácido esteárico de la monocapa en la subfase alcohólica, que se potencia a los valores más altos de temperatura y de concentración de etanol en la subfase.

\section{BIBLIOGRAFIA}

1. Becher, P. (Ed).-“Encyclopedia of Emulsion Technology, Vol. 2. Applications."-Dekker, New York, 1986.

2. Krog, N., and Lauridsen, J.-"Food Emulsifiers and their Associations with Water" in "Food emulsions."- S. Friberg (Ed).-Dekker, New York, 1976.

3. Lauridsen, J.-"Food emilsifiers: surface activity, edibility, manufacture, composition and application".- J. Am Oil Chemists' Soc. 53 (1976) 400-407.

4. Charalambous, G., and Doxatakis, G. (Ed).-"Food Emulsifiers: Chemistry, Technology, Functional Properties and Applications."Elsevier, Amsterdam, 1989

5. Dickinson, E., and Stainsby, G. (Ed).-"Advances in Food Emulsions and Foams".- Elsevier, Barking (Ingl.), 1988.

6. Krog, N.J.; Riison., T.H., and Larson, K.-"Applications in the Food Industry" in "Encyclopedia of Emulsion Technology."- P. Becher (Ed), Dekker, New York, 1985.

7. Mc Intyre, R.T.-"Fat-based emulsifiers" in "Fatty Acids in Industry."R.W. Johnson and E. Fritz (Eds).- Marcel Dekker, Inc, New York, 1989.

8. Buhaenko, M.R.; Grundy, M.J.; Richardson, R.M.-"Surface rheology of spread monolayers."- Thin Solid Film 159 (1988) 171189.

9. Miyano, K.; Abraham, B.; Ketterson, J., and Xu, S.-"The phases of insoluble monolayers: Comparison between the surface pressuremolecular area. Diagram and the shear modulus measurements."-J. Chem. Phys. 7 (1983) 4776-4777.
10. Boyd, J.V.; Parkinson, C., and Sherman, P.-"Factors affecting emulsion stability and the HLB (hydrophilic-lipophilic balance) concept."- J. Colloid Interface Sci 41 (1972) 359-370.

11. Dickinson, E., and Stainsby, G.-"Progress in the formulation of food emulsions and foams."- Food Technol. 41 (9) (1987) 7481

12. Larsson, K.-“Stability of emulsions formed by polar lipids."- Prog. Chem. Fats Other Lipids 16 (1978) 163-169.

13. Larsson, K.-"Lipid Monolayers" in "Surface and Colloid Science".- Vol. 6, P. 261-285.-E. Matijevic (Ed).-Wiley, New York, (1973).

14. Ogino, K., and Onishi, M.-“"Interfacial action of natural surfactant in oil/water systems".- J. Colloid Interface Sci. 83 (1981) 18.

15. Albrecht, D.; Grucer, H., and Sackmann, E.-"Polymorphism of phospholipid monolayers".- J. Physique 39 (1978) 301-313.

16. Lundquist, M.-"Molecular arrangement in condensed monolayer phases".- Prog.- Chem. Fats Other Lipids 16 (1978) 101-124.

17. Baret, J.F.; Bois, A.G.: Dupin, J.J., and Firpo, J.L-"The liquid expanded and the liquid condensed phases in amphiphile monolayers are separated by a second order transition."- J. Colloid Interface Scl. 86 (1982) 370-376.

18. Marcelja, S.-"Chain ordering in liquid crystals. II. Structure of bilayer membranes."-Biochim. Biophys. Acta 367 (1974) 165-176.

19. Pekalski, A.-"Phase diagrams for a model of a lipid monolayer".Eur. Biophys. J. 16 (1988) 39-44.

20. Ries, H.E. and Kimball, W.A.-"Structure of fatty-acid monolayers and mechanism for collapse".- 2nd International Congress Surf. Act., Vol I (1956) 75-84

21. Llopis, J.; España, F.; Sainz, J.L., y Albert, A.-“Monocapas de polipéptidos que contienen azufre".- An. Quim. 69 (1974) 12291238.

22. Gaines, G.L.-“Insoluble Monolayers at Liquid-Gas Interfaces".Intercience Publ., New York, 1966.

23. Dickinson, E.-"Effect of atractive forces on monolayer permeation and evaporation resistance".- J. Chem. Soc. Faraday Tran. II 74 (1978) 821-825

24. Gershfeld, N.-"The liquid condensed/liquid expanded transition in lipid films: a critical analysis of the film balance experiments".J. Colloid Interface Sci. 58 (1982) 28-40.

25. Gaines, G.-"The thermodinamic equation of state for insoluble monolayers. III. Mixed monolayers".- J. Colloid Interface Sci 85 (1982) $16-18$

26. Bilkadi, Z., and Neuman, R.-"Effect of humidity on desorption of fatty acid monolayers at constant area."- J. Colloid Interface Sci. 85 (1981) $480-489$

27. De la Fuente Feria, J_-“Estudio de Monocapas de Acidos Grasos sobre Disoluciones que Contienen Grupos Alcohólicos Presentes en Formulaciones alimentarias."- Tesis de Licenciatura, Universidad de Sevilla, 1989.

28. Garti, N., and Sato, K. (Eds).-"Cristallization and Polymorphism of Fats and Fatty Acids."- Dekker, New York, 1988.

(Recibido: Junio 1990) 